\title{
Demand side management of heat in smart homes: Living-lab experiments
}

\author{
Christensen, Morten Herget; Li, Rongling; Pinson, Pierre
}

Published in:

Energy

Link to article, DOI:

10.1016/j.energy.2020.116993

Publication date:

2020

Document Version

Peer reviewed version

Link back to DTU Orbit

Citation (APA):

Christensen, M. H., Li, R., \& Pinson, P. (2020). Demand side management of heat in smart homes: Living-lab experiments. Energy, 195, [116993]. https://doi.org/10.1016/j.energy.2020.116993

\section{General rights}

Copyright and moral rights for the publications made accessible in the public portal are retained by the authors and/or other copyright owners and it is a condition of accessing publications that users recognise and abide by the legal requirements associated with these rights.

- Users may download and print one copy of any publication from the public portal for the purpose of private study or research.

- You may not further distribute the material or use it for any profit-making activity or commercial gain

- You may freely distribute the URL identifying the publication in the public portal

If you believe that this document breaches copyright please contact us providing details, and we will remove access to the work immediately and investigate your claim. 


\section{Journal Pre-proof}

Demand side management of heat in smart homes: Living-lab experiments

Morten Herget Christensen, Rongling Li, Pierre Pinson

PII: S0360-5442(20)30100-6

DOI: $\quad$ https://doi.org/10.1016/j.energy.2020.116993

Reference: EGY 116993

To appear in: Energy

Received Date: 29 October 2019

Revised Date: 13 January 2020

Accepted Date: 16 January 2020

Please cite this article as: Christensen MH, Li R, Pinson P, Demand side management of heat in smart homes: Living-lab experiments, Energy (2020), doi: https://doi.org/10.1016/j.energy.2020.116993.

This is a PDF file of an article that has undergone enhancements after acceptance, such as the addition of a cover page and metadata, and formatting for readability, but it is not yet the definitive version of record. This version will undergo additional copyediting, typesetting and review before it is published in its final form, but we are providing this version to give early visibility of the article. Please note that, during the production process, errors may be discovered which could affect the content, and all legal disclaimers that apply to the journal pertain.

(ㄷ 2020 Published by Elsevier Ltd. 


\title{
Demand side management of heat in smart homes: Living-lab experiments
}

\author{
Morten Herget Christensen ${ }^{\mathrm{a}, *}$, Rongling Li ${ }^{\mathrm{b}}$, Pierre Pinson ${ }^{\mathrm{a}}$ \\ ${ }^{a}$ Center for Electric Power and Energy, Department of Electrical Engineering, Technical University of \\ Denmark, 2800 Kgs. Lyngby, Denmark \\ ${ }^{b}$ Section for Energy and Services, Department of Civil Engineering, Technical University of Denmark, \\ 2800 Kgs. Lyngby, Denmark
}

\begin{abstract}
In smart energy systems the role of the building is transformed from being a passive consumer into an energy flexibility provider. Buildings are expected to have flexible energy demand to provide services to energy grids. In this paper we present an experimental study conducted in a multi-storey residential apartment building. In the experiments supervisory control of individual room temperatures was applied to provide direct demand response for district heating grids. Control signals were applied to the individual floor heating systems in about 90 rooms in order to reduce heating demand in peak load hours. The results show that there is a significant potential for flexible energy consumption in homes based on smart home systems. It was found that when using a simple time based penalty signal, on average, the peak-hour energy consumption was reduced by $85 \%$ with little impact on overall energy consumption and indoor temperature.
\end{abstract}

Keywords: Demand side management, Energy flexibility, Floor heating, District heating, Living lab, Experiments

\section{Introduction}

The need for changes in the energy systems is apparent and acute, as the negative impacts of an economy based on the burning of fossil fuels has long been a well established fact [1]. The transition towards cleaner alternatives has gained momentum [2] as investments in renewable energy production are rising at unprecedented rates. In Denmark, the capital city of Copenhagen has committed to carbon neutrality by 2025. A significant share of the energy is used to provide heat to buildings [3] especially in colder climates. On average, residential energy demand accounts for $26 \%$ of total final energy demand in EU-28 countries and the share of space heating energy demand is $65 \%$ of the energy demand in the residential

\footnotetext{
*I am corresponding author
}

Email addresses: mhchris@elektro.dtu.dk (Morten Herget Christensen), liron@byg.dtu.dk (Rongling Li), ppin@elektro.dtu.dk (Pierre Pinson) 
sector [4]. District heating has been in a transition from the use of coal and natural gas towards renewable energy sources such as biomass [5], while electricity production from clean sources is steadily increasing, mainly from wind turbines and solar photovoltaics (PV). The potential benefits of sector-coupling have led to intense studies of integrated energy systems approaches at all levels including markets [6] and districts [7]. The 4th generation district heating systems [8] highlight the possibilities as cross-sector optimization and planning between electricity and heating systems. Smart energy systems [9] expands on this concept to take a holistic approach including electrofuels, energy efficiency and storage eventually showing a cost effective path to $100 \%$ renewable systems [10].

More than 30\% of all energy use takes place in buildings [3]. Thus, stricter requirements in building codes as well as technological advances in Information and Communication Technologies (ICT) will lead to increased automation levels in the built environment [11. The increased automation levels in the smart buildings bring the opportunity of using buildings to provide flexibility in energy consumption as shown in [12], where the focus is on the control of smart buildings for auxiliary services provision. These advances are complimented by new consumer-centric market models aiming at activating the potential for flexible consumption [13]. The concept of flexible consumption for modern low energy buildings have been examined [14] and expanded for district heating networks [15]. Applications of the built environment and district heating networks as a source of thermal storage is reviewed in [16], highlighting that the regulatory and economic framework conditions are challenging commercial realizations.

Price based demand response has been shown to be effective [17] in providing services to electrical power systems. Previous work have shown how advanced control methods such as model predictive control can be applied to optimize the indoor environment [18], or to make rooms [19] or buildings price responsive [20]. However, the majority of the studies are simulation-based. A practical implementation of these methods offer challenges that are not fully captured by simulation based studies [21]. In [22] the impact of external disturbances on the flexibility potential of low-energy buildings is studied, but the results are based on simulations and assumptions on e.g. user behavior. Real-life applications are still lacking in order to verify the simulation-based results. A study [23] applied demand side management to 28 homes on a district heating grid in the UK in order to increase the load factor of the system, however the systems here are radiator-based systems and single family homes and the results do not readily transfer to e.g. dense urban environments with lowenergy multi-storey buildings. To the best of our knowledge, using multi-story residential buildings with control of individual room temperatures to provide direct demand response for district heating grids have not been implemented. In addition, the definition of energy flexibility and thus methods for quantifying flexibility of buildings are not well established [24. Recent work has attempted to provide coherent definitions of flexibility function and flexibility indicators suitable for use in demand response [25]. These definitions have not been implemented and tested in real life experiments.

A recent review of field studies on power grid integration of residential thermal energy storage concluded that existing field tests did not meet the flexibility challenges of smart grids with high share of renewable generation [16]. In comparison with the control of power grids, 
the control of district heating grids has lower frequency due to thermal inertia in buildings and large lags in heat transmission and distribution networks, e.g. depending on the size of the network, the effect of the change of supply water temperature made at heat production plant can only be seen a few hours later on the demand side, i.e. in buildings. Therefore, it is feasible to implement heating demand management for district heating grids. Although field tests of activating heating demand flexibility for district heating load management are still limited, promising results are shown in these tests. Liu et.al reported a $30 \%$ energy saving by adjusting and metering household heating systems in a large scale pilot [26]. Demand side management (DSM) demonstrations in two Finnish buildings with concrete structures shown a heat load reduction of $20-25 \%$ for a duration of 2-3 hours [27], however their approach can disturb thermal comfort as it does not respect the differing expectation of comfort in individual rooms. A field trial of demand shifting technology on a DH network was conducted in the UK with reduced peak demand and a slight increase (3\%) in heating consumption [23]. To gain insight into the controllability of space heating demand, the management of heating demand for reducing fossil fuel usage and CO2 emission in district heating systems, we conducted field tests in 13 homes equipped with ICT technologies in Copenhagen, Denmark in the heating season of 2018/19. The study seeks to bring more understanding of the potentials of the built environment in the renewable transition. Building on the flexibility indicator of [25] we show how these can be used to evaluate the flexibility potential of a number of newly built apartments in Copenhagen.

The remainder of this paper is organized as follows: In section 2 the building under investigation and its context in the living lab in the Nordhavn district is presented. Section 3 details how the flexibility experiment was designed and the methodology used to quantify the flexibility services and indoor temperature effects. In Section 4 the results of the study is presented.

\section{Experiment setup}

As a part of the project EnergyLab Nordhavn [28], which is a demonstration and development project on future integrated energy systems, located in Copenhagen, Denmark, we equipped a number of apartments with home automation systems including indoor environment sensors, floor heating actuators and energy meters. The equipment was installed so that the apartments would become living labs. In this section the capabilities of the living labs are presented.

\subsection{Building description}

The particular building that houses the apartments used in this study is located in the district of Nordhavn shown on Figure 1. The building is a multi-story residential building with 72 apartments and a total heated area of approximately $7000 \mathrm{~m}^{2}$. The apartments range from 47 to $209 \mathrm{~m}^{2}$ and has from 2 to 10 heated rooms including living room/kitchen, bedrooms, bathrooms, toilets and depots [29]. The building was constructed in 2017 in accordance with energy class BR2020 of the danish building code BR15[30]. The total energy use for heating, ventilation, cooling and domestic hot water for such buildings is 


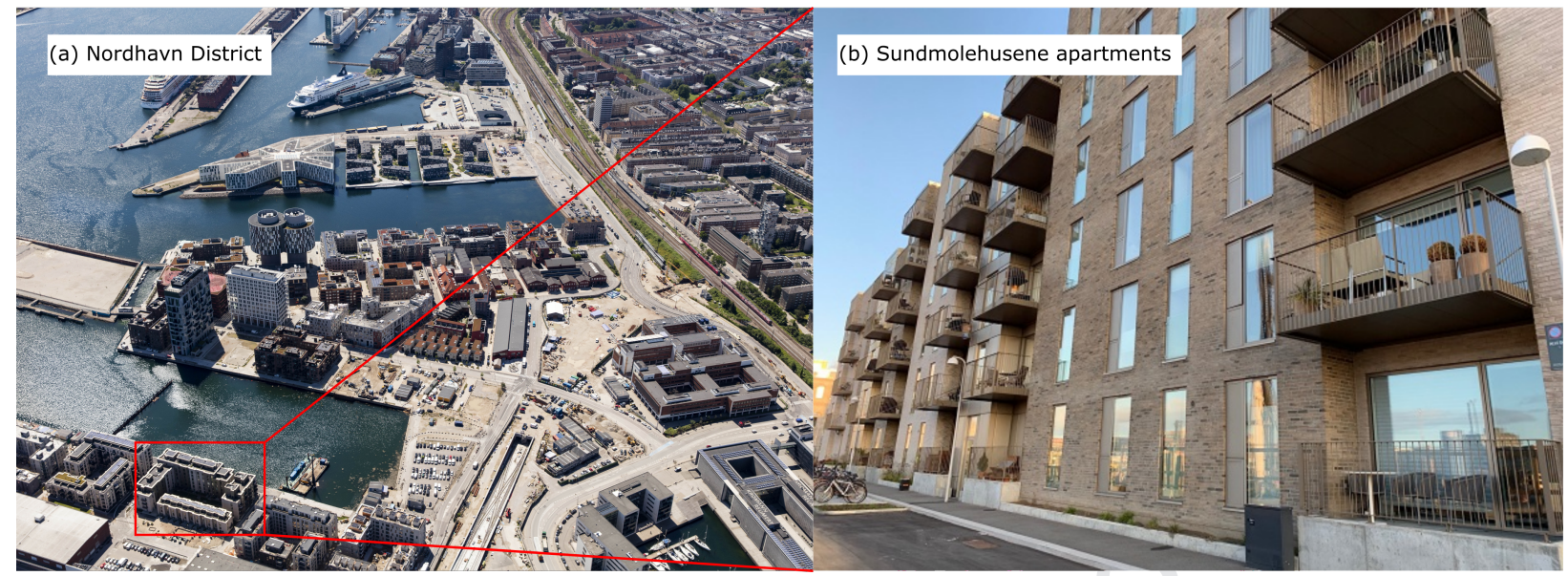

Figure 1: (a) An overview of the newly built Nordhavn district (b) Facades of the Sundmolehusene apartments

limited to $20 \mathrm{kWh}$ per square meter heated floor area per year [30]. The building is highly insulated and very air tight. Mechanical ventilation systems have heat recovery in order to keep heat loss to a minimum. These apartments have heavy concrete load bearing walls with heavy thermal mass.

\subsection{Apartment heating system}

The apartments are primarily heated by radiant floor heating systems where warm water is circulated in pipes that are cast in a light layer of concrete that sits on top of an insulation layer in the floor. The light concrete layer with the heating pipes sits under wooden floors. The flow-rate of the warm water, and the resulting heat delivered to the apartments, is controlled by local thermostatic controllers that open and close the valves supplying the independent heating loops as can be seen from the principle diagram and typical apartment floor plan in Figure 3. The details are based on the design of the experiment building as it was constructed in Copenhagen by commercial contractors. In modern apartment buildings in the Danish construction it is customary to include thermostatic control of individual rooms including bathrooms. The opening and closing of valves is based on the current temperature in individual rooms as well as the user-adjustable set-point for that room. The warm water is supplied from individual shunt loops that regulates the temperature supplied to the apartments to a maximum of 35 degrees Celsius. The local control system loop is shown in block diagram form in Figure 2. The shunt loops are supplied by a mixing loop located in the heating substation (not shown). The substation is supplied by district heating. The heat delivered to each apartment is individually metered through heat meters that measure the volumetric flow, forward and return temperatures of the water supplied to the shunt loops.

\subsection{Related work in Nordhavn living labs}

The buildings in Nordhavn has attracted attention for research due to the abundance of data source from the buildings. In [22] the impact of weather and users for a building in the 


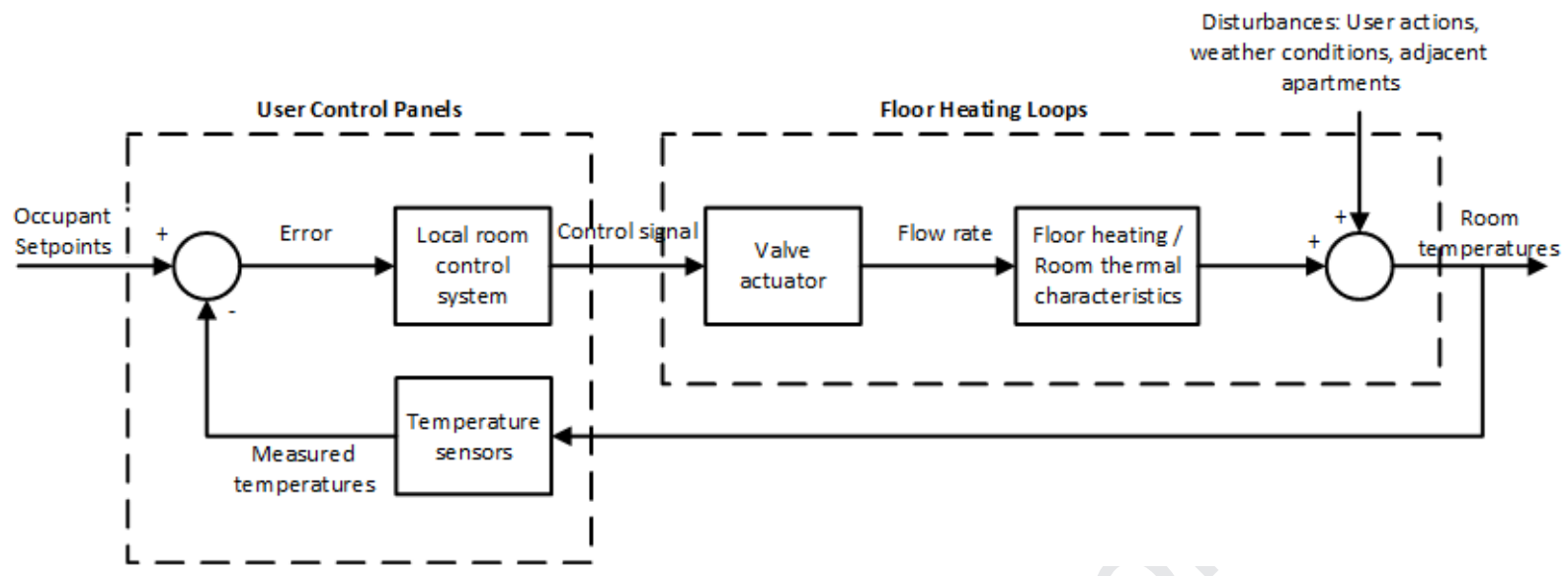

Figure 2: Local control systems of the Smart homes controlling the indoor temperature
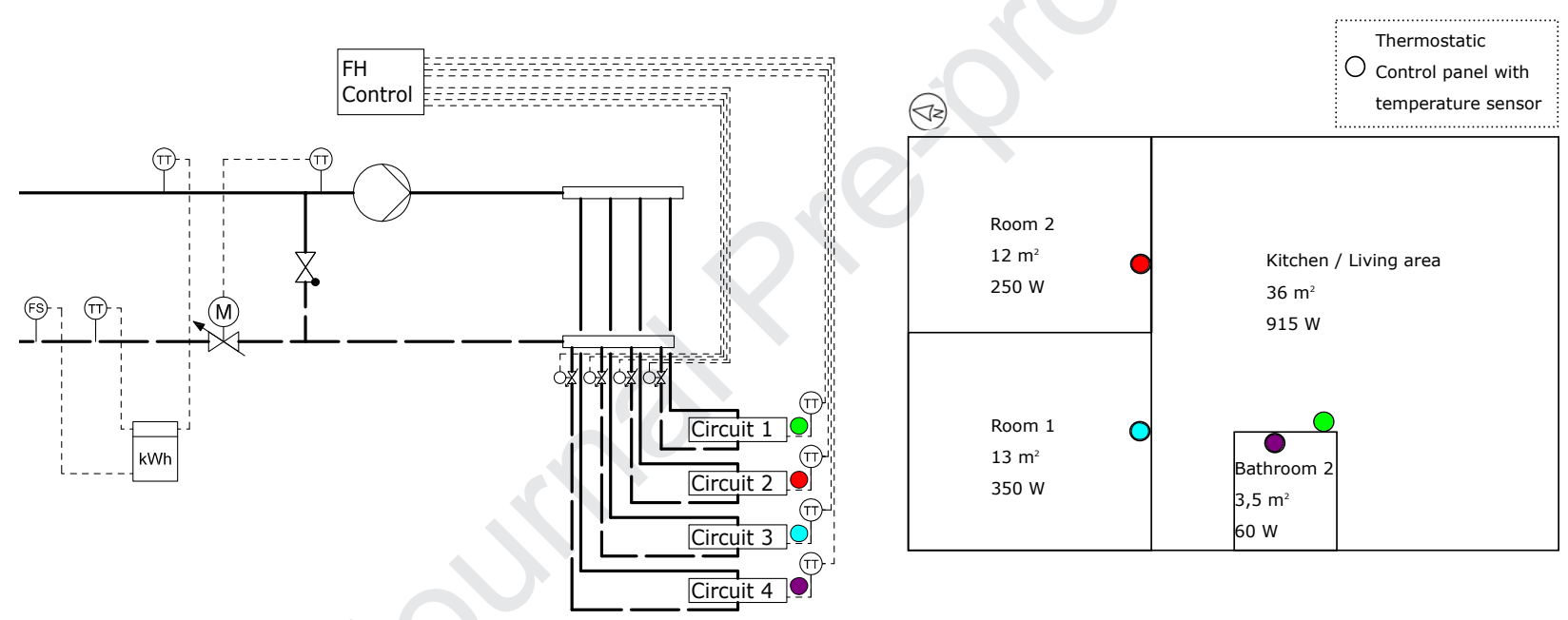

Figure 3: Example of details of floor heating systems: Principal drawing and corresponding heating loop distribution

Nordhavn district is studied based on simulations. The results in that study indicate that the buildings have very high thermal inertia and that the thermal flexibility, which is defined as the time before the room temperature drops below 20 degree $\mathrm{C}$ is more than 10 hours. Similarly [14 reports that after an 8 hour period without heat supply to space heating, the building has less than 1 degree $\mathrm{C}$ reduction in indoor temperature. The high thermal inertia is not symmetric towards increases and decreases in indoor temperature. This is obvious when you consider that these type of buildings have active heating systems but in general no active cooling systems. When cooling the building the process relies mainly on the exchange of energy between the highly insulated building and the surroundings. The implication is that the risk of overheating is higher than the opposite. In [31] data from selected apartments in the building is analyzed to give insights into the user preferences based on their set-points and interviews with the residents. The results show a great variability in 
the usage of the systems that will ultimately have a significant influence on the potential for flexible consumption. The high thermal inertia assumption is investigated in detail in [32] where data from temperature sensors casts into the walls and ceilings of the apartments is analyzed.

\subsection{Information and communication technology}

The local control systems in the apartments are off-the-shelves components that are based on the KNX protocol and architecture. The KNX protocol [33] is used for Direct Digital Control (DDC), a form of de-centralized distributed control where individual sensors and actuators are connected by a digital communication bus. The individual components then communicate directly with each other, exchanging a minimum of necessary information. The data from the local control systems is continuously transferred by encrypted Internet Protocol interface to the data management system (DMS) at the Technical University of Denmark where data is translated to Message Queuing Telemetry Transport (MQTT) messages as shown in Figure 4. Sensors and actuators are located physically in the individual apartments, the data and command signals are communicated to the data management system through the open source protocol MQTT. The protocol works on top of TCP/IP in the application layer of the internet protocol suite. Several open source implementation of MQTT are available i.e. Mosquitto [34]. The KNX messages are translated to MQTT format by a software gateway in the data management system. The MQTT messages can in this way be subscribed to by logging services such as the data warehouse (PowerLabDK) in the project, but also for control applications and on-line monitoring. A major limitation of this setup, is that in the case of networking errors data will be lost since there is no local logging of data. A major benefit is that the systems employed is identical to those that are installed in real buildings under commercial terms and thus the solutions that are developed using these systems are implementable in other buildings with KNX or similar control systems installed.

\subsection{Data description}

The data that is used in the study includes the energy consumption and heating power of the floor heating systems in the individual apartments along with the indoor temperatures and set-points of the controlled rooms in the apartments. The data is collected from the local systems from a heat meter that measures the volumetric flow, forward and return temperatures of the water supplied to the floor heating systems. From these measurements, the meter also provides the heating power, accumulated energy use and total volume of water used. The thermostatic control systems include (1) local operating panels that show the room temperature and registers set-points from user input, and (2) hysteresis controllers that send control values to valve actuators.

\section{Methodology}

In winter 2018/2019 we carried out the experiments in selected apartments in the buildings. The main experiment period was March 2019 in the heating season which is typically 


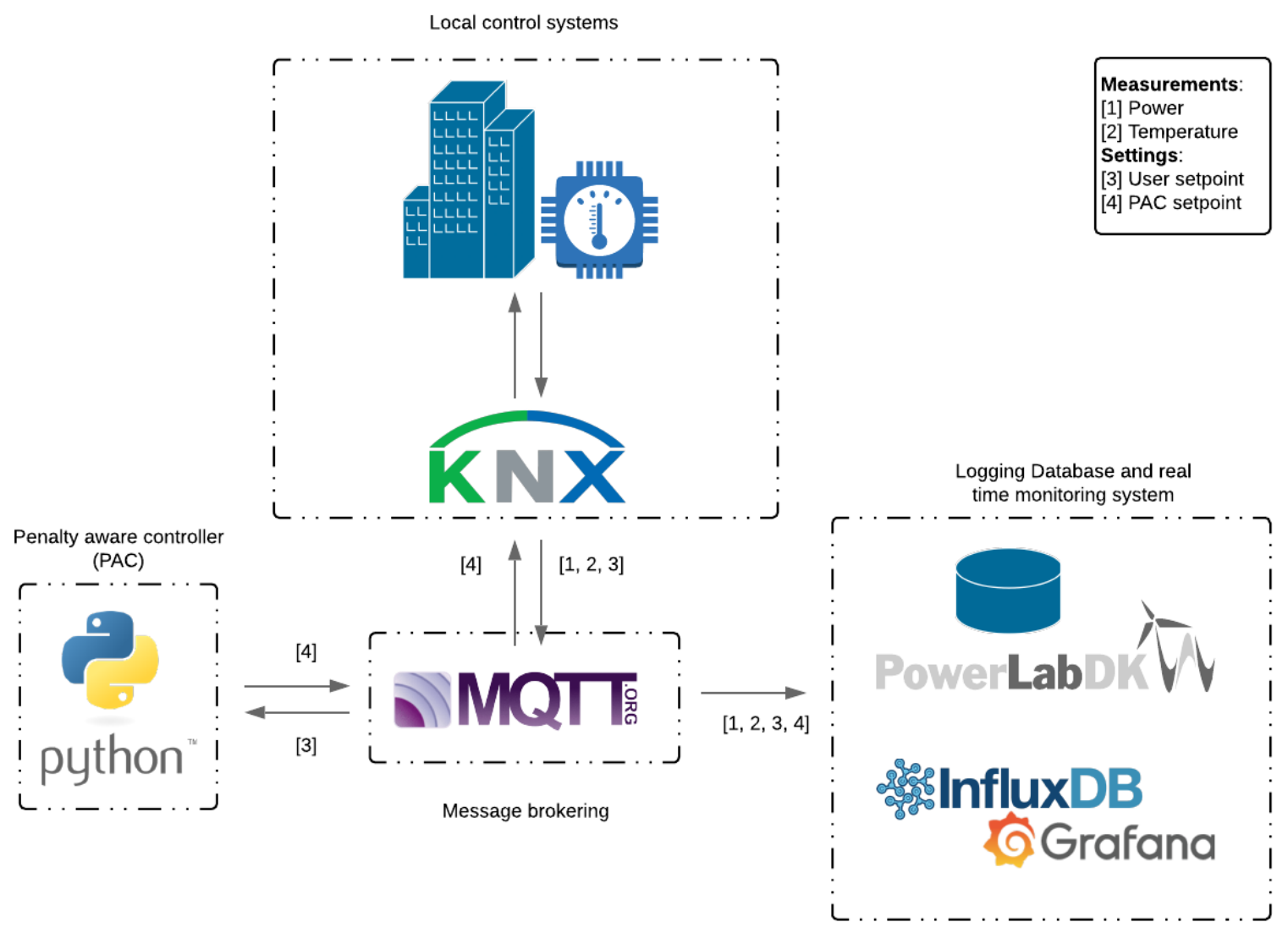

Figure 4: Data flows connecting the smart homes with the data management systems at PowerLab.dk and the penalty aware controller 
Table 1: Data specification

\begin{tabular}{lllll}
\hline Data source description & Symbol & Unit & Source & [ID] \\
\hline $\begin{array}{l}\text { Heat meter } \\
\text { Forward temperature of floor }\end{array} T_{i}^{\mathrm{f}}$ & Degree Celsius & Direct measurement \\
heating shunt in apartment $i$ at & & & \\
time $t$ & & & \\
$\begin{array}{l}\text { Return temperature of floor heat- } \\
\text { ing shunt in apartment } i \text { at time }\end{array}$ & $T_{i}^{\mathrm{r}}$ & Degree Celsius & Direct measurement \\
$t$ & & & \\
$\begin{array}{l}\text { Temperature difference between } \\
\text { forward and return in apartment }\end{array}$ & $\Delta \Theta_{i}$ & Kelvin & $T_{i}^{\mathrm{f}}-T_{i}^{\mathrm{r}}$ \\
$i$ at time $t$ & & \\
$\begin{array}{l}\text { Volumetric flow in floor heating } \\
\text { shunt of apartment } i \text { at time } t\end{array}$ & $\dot{V}_{i}$ & $\mathrm{~m}^{3} / \mathrm{h}$ & Direct measurement \\
$\begin{array}{l}\text { Total Volume in floor heating } \\
\text { shunt of apartment } i\end{array}$ & $V_{i}$ & $\mathrm{~m}^{3} / \mathrm{h}$ & $\int \dot{V}_{i}$ \\
$\begin{array}{l}\text { Instantaneous power of apart- } \\
\text { ment } i \text { at time } t,\end{array}$ & $P_{i}$ & $\mathrm{~W}$ & $\Delta \Theta_{i} F_{i} c$ \\
$\begin{array}{l}\text { Meter reading, accumulated en- } \\
\text { ergy use of apartment } i \text { at time }\end{array}$ & $E_{i}$ & $\mathrm{kWh}$ & $V_{i} \Delta \Theta_{i} c$ \\
$t$ & & & \\
$\begin{array}{l}\text { Thermostatic control panel } \\
\text { Room temperature in room } j \text { in } \\
\text { apartment } i \text { at time } t\end{array}$ & $T_{i, j}$ & Degree Celsius & Direct measurement & [\#2] \\
User set-point in room $j$ in apart- & $T_{i, j}^{\text {ref }}$ \\
ment $i$ at time $t$ & Degree Celsius & Direct measurement & [\#3]
\end{tabular}

from early October to late April. The period was the earliest possible respecting project constraints and coordination with other project participants. The experimental setup evolved from the findings of the earliest tests. The experiment protocols were updated based on feedback from the residents.

\subsection{Penalty signal design}

The objective of the demonstration is to show that a significant amount of heating load can be shifted from the peak hours. To achieve the objective, initially, we experimented with a pre-heating strategy, that would raise the temperature in the hours before the reduction period. Early tests showed that pre-heating was unnecessary due to the high thermal inertia of the building. As a result, we gradually reduced the pre-heating, from a few hours to ultimately a pure peak-shaving where no pre-heating was applied. The resulting penalty signal with and without pre-heating are shown in Figure 5. A penalty of \pm 1 corresponds to a desired reduction of $\pm 100 \%$ of the power output of the floor heating system. 

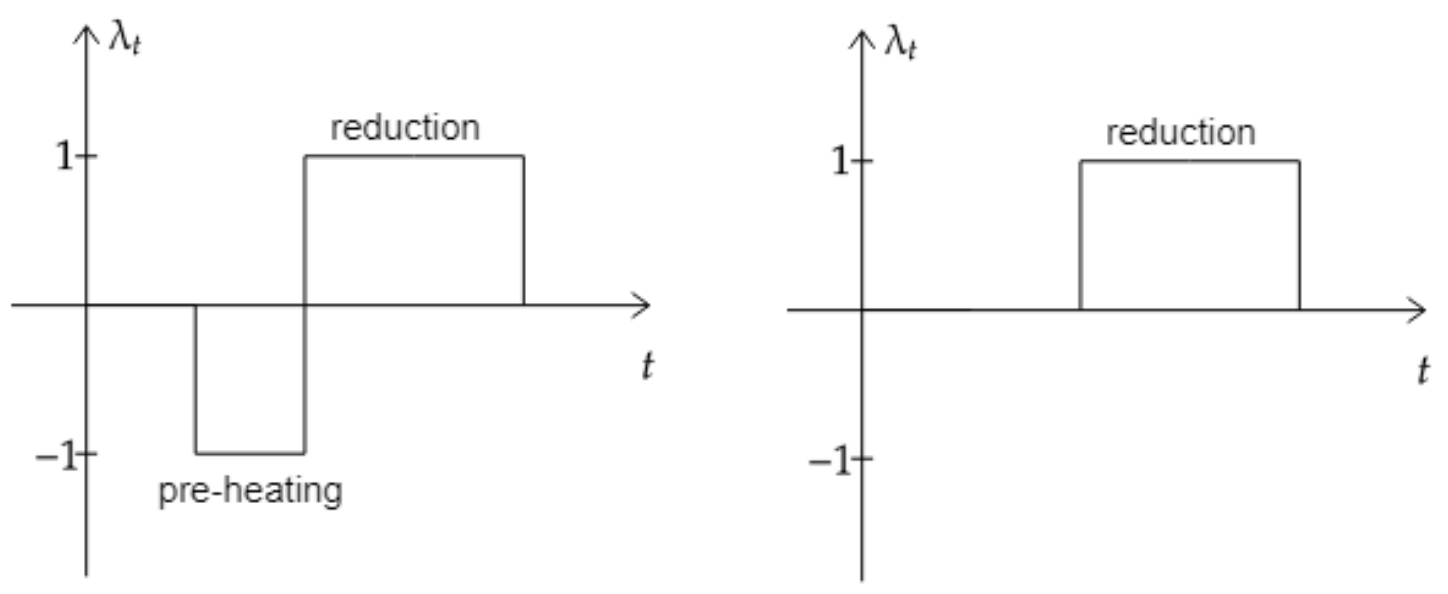

Figure 5: Penalty signals with and without pre-heating of the building

\subsection{Penalty signal implementation}

In the apartments local automatic controllers maintain the room temperature. We implemented Algorithm 1 as a supervisory control that send new set-points to the local systems. This lets the local control parameters to remain as they were when the systems where installed and commissioned. The algorithm reads set-points from the apartment and sends new corrected set-points for the peek-hour period to the apartment controllers. At all times the current power and temperature measurements are recorded. A technical limitation in the KNX - DMS hybrid control is that there is only a few possibilities to interact with the floor heating system. The historical data of temperature set-points for individual rooms are used as user preferred set-points. During the peak hour reduction period, a lower set-point is sent to the local control at 5 minute intervals. There are two situations in which the penalty signal will not effective in effecting heating systems as expected: [i] room under-heated, where the heating will be fully on regardless of the penalty signal; [ii] room overheated, where the heating will be fully off regardless of the penalty signal. Practical experience from the early tests of the system and dialogue with the users revealed that the loss of control was experienced negatively by the residents. They had especially strong objections against loss of control for the bathroom in the morning hours. As a consequence, we omitted control of bathrooms and toilets from the experiment. In Algorithm 1 the timetables were chosen based on a series of empirical sequential tests to determine suitable lengths of pre-heating and ramping rate of re-activation. The methodology we employed investigated an initial hypothesis of the necessity of pre-heating the apartments in order to prolong the period of reduced heating while respecting temperature limits. The six-hour reduction period was requested by HOFOR, the greater Copenhagen utility company that supplies the district heating to reduce morning peak demand. The algorithm was implemented in Python and run on a linux machine using CRON jobs to schedule the execution time of the script. The source code is available at Zenodo: https://zenodo.org/record/3600114 [35] 


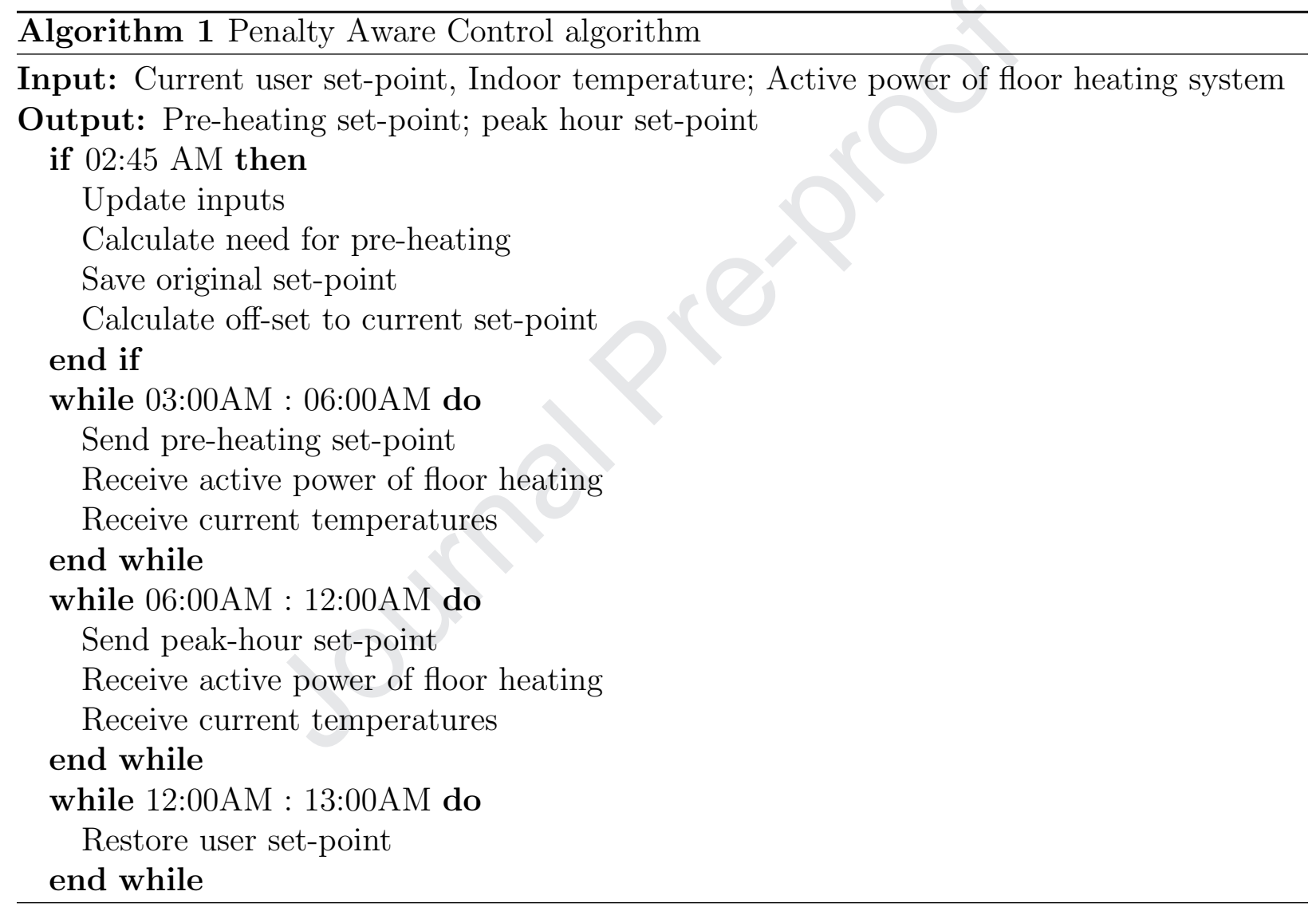




\subsection{Experiment assessment}

To quantify the effectiveness of the experiment and penalty signal in generating a response from the apartments, it is necessary to try to account for the exogenous variables that will impact the consumption. For instance, outdoor temperature and solar irradiance are known to correlate with energy use for buildings. This makes it harder to quantify actual effects of the control strategy using a naive deviation penalty. A suitable reference point for comparison is called a baseline and is often established by applying normalization techniques that seek to remove the effect of the exogenous variables, such as in [36] [37] [38]. An alternative to the normalization approaches is to take an approach similar to that employed in clinical trials in pharmaceutical research, where the participants in the trial are divided into treatment and control groups. This approach was used in [17]. Ideally this should be conducted in a double-blind setup so that neither the person administering the treatment or the subject receiving the treatment knows whether or not the real treatment or a inactive "placebo" treatment is given.

In this study by analogy we defined treatment and control groups of apartments, where the treatment group is subjected to the penalty aware controller and the control group is not. The apartment characteristics of the two groups are shown in Table 2. Since the local controls are automated they are not susceptible to the placebo effect and no placebo or double blind study is deemed necessary.

Table 2: Apartment characteristics

\begin{tabular}{lrr}
\hline Apartment Identifier & Size & Heating power \\
\hline Control group & $75.4 \mathrm{~m}^{2}$ & $1771 \mathrm{~W}$ \\
A35 & $66.1 \mathrm{~m}^{2}$ & $1523 \mathrm{~W}$ \\
A36 & $75.4 \mathrm{~m}^{2}$ & $1771 \mathrm{~W}$ \\
A39 & $22.7 \mathrm{~m}^{2}$ & $800 \mathrm{~W}$ \\
B11 & $46.7 \mathrm{~m}^{2}$ & $1497 \mathrm{~W}$ \\
B13 & $36.7 \mathrm{~m}^{2}$ & $968 \mathrm{~W}$ \\
B18 & & \\
& $323 \mathrm{~m}^{2}$ & $8330 \mathrm{~W}$ \\
\hline Group total & & \\
\hline Experiment group & $75.4 \mathrm{~m}^{2}$ & $1771 \mathrm{~W}$ \\
A33 & $66.1 \mathrm{~m}^{2}$ & $1523 \mathrm{~W}$ \\
A40 & $87.7 \mathrm{~m}^{2}$ & $2310 \mathrm{~W}$ \\
A42 & $36.7 \mathrm{~m}^{2}$ & $968 \mathrm{~W}$ \\
B14 & $46.7 \mathrm{~m}^{2}$ & $1497 \mathrm{~W}$ \\
B15 & $46.7 \mathrm{~m}^{2}$ & $1497 \mathrm{~W}$ \\
B17 & $46.7 \mathrm{~m}^{2}$ & $1497 \mathrm{~W}$ \\
B19 & $406 \mathrm{~m}^{2}$ & $11063 \mathrm{~W}$ \\
\hline Group total & &
\end{tabular}




\subsubsection{Energy flexibility indices}

We propose an energy flexibility index based on the work of Annex 67 of the International Energy Agency (IEA) [39] to quantify the achieved energy flexibility. In the Annex 67 of the IEA, a energy flexible building is defined to have "the ability to manage its demand and generation according to local climate conditions, user needs and grid requirements". Clearly the term has different usage according to domain and context. The authors of [25], building upon the definition from Annex 67, propose a method for estimation of a flexibility index that we adopt in this study, with

$$
\mathrm{FI}=1-\frac{C^{1}}{C^{0}}
$$

where

$$
C^{0}=\sum_{t=0}^{N} \lambda_{t} u_{t}^{0}
$$

is the penalty-ignorant response under penalty $\lambda_{t}$ with energy consumption $u_{t}^{0}$, and where

$$
C^{1}=\sum_{t=0}^{N} \lambda_{t} u_{t}^{1}
$$

is the penalty-aware response under the same penalty $\lambda_{t}$ with energy consumption $u_{t}^{1}$.

In the case where only positive unit steps of penalties are employed the cost are in fact identical to the energy consumption in the penalty period. This definition works well for simulation based studies where the building under investigation can be subjected to identical conditions of e.g. weather and usage, allowing to establish the reference case $C^{0}$ (the penalty-ignorant response) with ease. For real buildings it is not possible to subject the building to identical conditions. For buildings with abundant historical data it might be possible to use normalization techniques to provide a good reference by e.g. training of regression models that account for the parameters that is known to have a significant influence on the energy consumption. For newer buildings with limited historical data, it might not be possible to provide a objective reference and the normalization technique used can become be a dominant factor in the results obtained.

\subsubsection{Impact on indoor temperatures}

The primary purpose of the delivery of heat to the apartments is not to provide flexibility but to ensure a comfortable living environment for the residents. In order to quantify the quality of the heating service provided by the flexible heating control strategies we use the lumped indoor temperature to compare the reference and the experiments. The lumped indoor temperature for an apartment is used instead of using individual temperatures from every room of the apartment. This is because the measurement of heating power is at the apartment level but not at the individual room level. The lumped temperature is the sum of all room temperatures scaled by the room size $A_{j}$ divided by the apartment size $A_{i}$ for 
room $j$ in each apartment $i$. We also take any redundant sensors in account by the factor $n_{j}$.

$$
\tilde{T}_{i}^{\mathrm{l}}=\sum_{j=1}^{N} \frac{T_{i, j} A_{j}}{n_{j} A_{i}}
$$

Another common measure of similarity between time-series is to calculate the Root Mean Square Error (RMSE) which for a single time series with $\mathrm{N}$ discrete steps and increments of $\mathrm{k}$ we define as

$$
\operatorname{RMSE}_{i, j}=\sqrt{\frac{1}{N} \sum_{k=1}^{N} e_{i, j}(k)^{2}}
$$

where

$$
e_{i, j}=T_{i, j}-\widetilde{T_{i, j}^{\mathrm{ref}}}
$$

Here we have used the historical median indoor temperature $\widetilde{T_{i, j}^{\text {ref }}}$ in place of the set-point which is normally the reference for the error. This is necessary since the set-point is being continuously overwritten as explained by Algorithm 1. Using (5) we define the lumped indoor RMSE scaled by the room sizes per apartment

$$
\widetilde{\mathrm{MSE}}_{i}^{1}=\sum_{j=1}^{N} \frac{\mathrm{RMSE}_{i, j} A_{j}}{n_{j} A_{i}}
$$

\section{Results}

In the following sections results from individual apartments as well as aggregated results of the total experiment are presented.

\subsection{Flexibility index}

We evaluate the flexibility response by calculating the flexibility index and by visual inspection of the aggregated power consumption time series for the two periods. When comparing the time series signals, shown in Figures 6 and 7 for the two groups we see that while the control group has little reaction, the power consumption during the 06:00 to 12:00 for all days is drastically lowered for the treatment group in the experiment period. It is only for the treatment group during the penalty hours in the experiment period that we see the very sharp drop to near zero consumption. In the reference period, Figure 6, both groups show no reaction during the penalty hours as expected, since no signal was send to the apartments. If we compare Figure 7 with Figure 6, we note that in Figure 7 the heating power in the hours from 12 to 6 is higher on some days. This is due to rebound effect as some heating demand was shifted to these hours after the penalty period. From Equation (1) we calculate the flexibility index. For the control group we find that there was a $6 \%$ reduction during the penalty hours when compared to the reference period. 


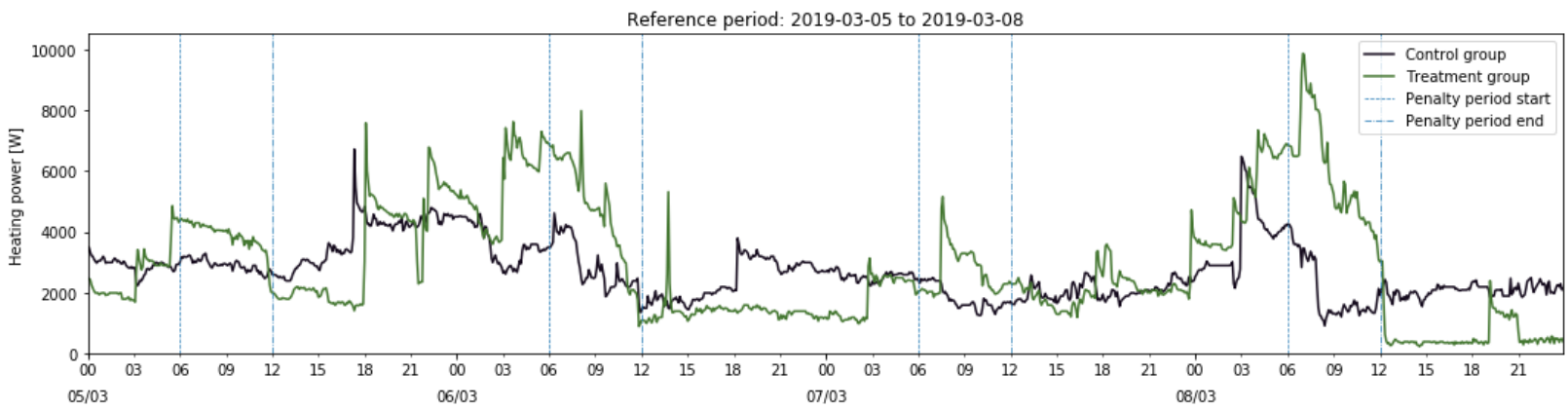

Figure 6: Aggregated heating power in apartments in control and treatment groups during the reference period

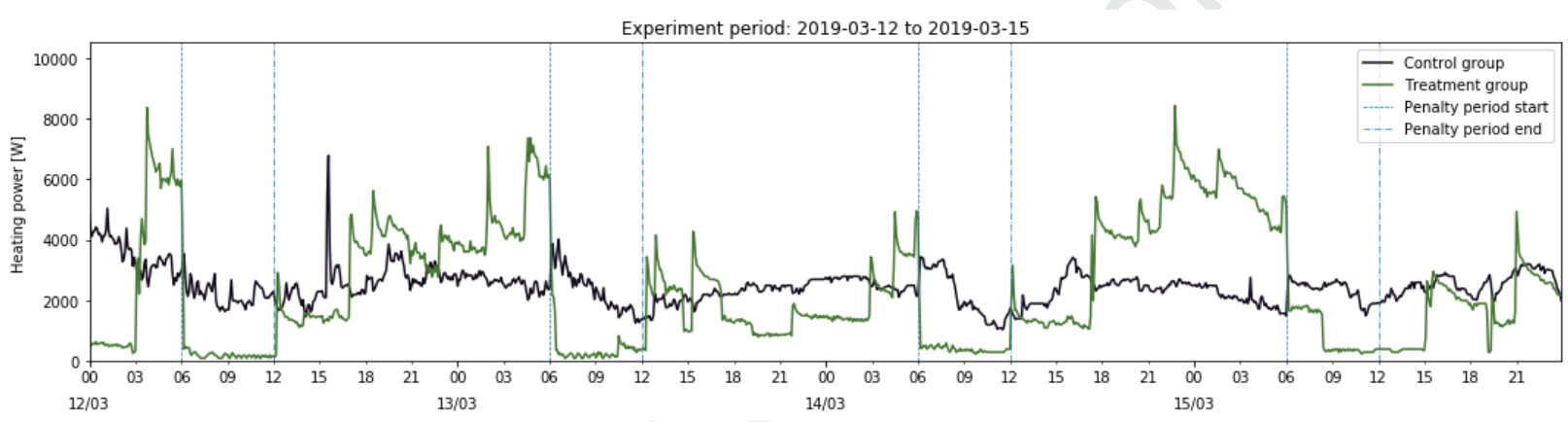

Figure 7: Aggregated heating power in apartments in control and treatment groups during the experiment period

For the treatment group the corresponding result was $85 \%$. The results of the individual apartments are reported in Table 3. In this study, careful observation of the system, e.g. valve control signals, circulation pump current was done in stead in order to verify that the results obtained are indeed caused by the controls implemented and not just a random phenomena. A more rigorous statistical approach would be to include hypothesis testing in order to give a statistical validation of the difference observed for the two groups.

The results thus confirm that the penalty aware controller can activate the flexibility potential. When we calculate the total energy consumption for the two groups during all hours, we find that for the control group the experiment period had a total energy consumption that was $7 \%$ less than the reference period. For the treatment group the result was a $20 \%$ reduction. From our result we thus do not see an increase in energy consumption as a result of the experiment. Upon closer inspection we see that the main part of the reduction is dominated by significant reduction in two apartments, B14 and B15 while a single apartment B19 actually had a large increase in total energy consumption. The result agrees with the intuition that, as we did not pre-heat the apartments the temperatures would be expected to be slightly lower on average and thus the total energy consumption would be expected to be slightly lower as well. 
Table 3: Energy consumption and flexibility indicators FI results

\begin{tabular}{|c|c|c|c|c|c|c|c|c|}
\hline \multicolumn{9}{|c|}{ Control group energy consumption during peak hours $06.00-12.00$ in $[\mathrm{kWh}]$} \\
\hline & & A35 & A36 & A39 & B11 & B13 & B18 & Total \\
\hline Reference period $C^{C 0}$ & & 7.0 & 11.0 & 7.0 & 5.0 & 16.0 & 22.0 & 68.0 \\
\hline Experiment period $C^{C}$ & & 6.0 & 8.0 & 8.0 & 5.0 & 23.0 & 14.0 & 64.0 \\
\hline$F I^{C}=1-\frac{C^{C 0}}{C^{C}}$ & & 0.14 & 0.27 & -0.14 & 0.0 & -0.44 & 0.36 & 0.06 \\
\hline \multicolumn{9}{|c|}{ Treatment group energy consumption during peak hours $06.00-12.00$ in $[\mathrm{kWh}]$} \\
\hline & A33 & A40 & A42 & B14 & B15 & B17 & B19 & Total \\
\hline Refe & 24.0 & 17.0 & 23.0 & 21.0 & 6.0 & 8.0 & 15. & 114.0 \\
\hline Experiment $C^{T}$ & 0.0 & 5.0 & 4.0 & 4.0 & 0.0 & 3.0 & 1.0 & 17.0 \\
\hline$F I^{T}=1-\frac{C^{T}}{C^{T 0}}$ & 1.0 & 0.71 & 0.83 & 0.81 & 1.0 & 0.63 & 0.93 & 0.85 \\
\hline
\end{tabular}

\subsection{Effects on the indoor temperature}

The effect of the flexibility demonstration on the indoor temperatures would be expected to have a lower indoor temperature as a result of the peak shaving without preheating. It is noted that it is not the case that all apartments have lower temperatures from the box plots in Figures 8 and 9. Besides heating supply, there are a number of other factors influence the indoor temperatures, e.g. solar irradiation, internal heat gains from appliances and people, mechanical ventilation and heat transfers between adjacent apartments. These dynamics are too complicated to fully account for within the scope of this study
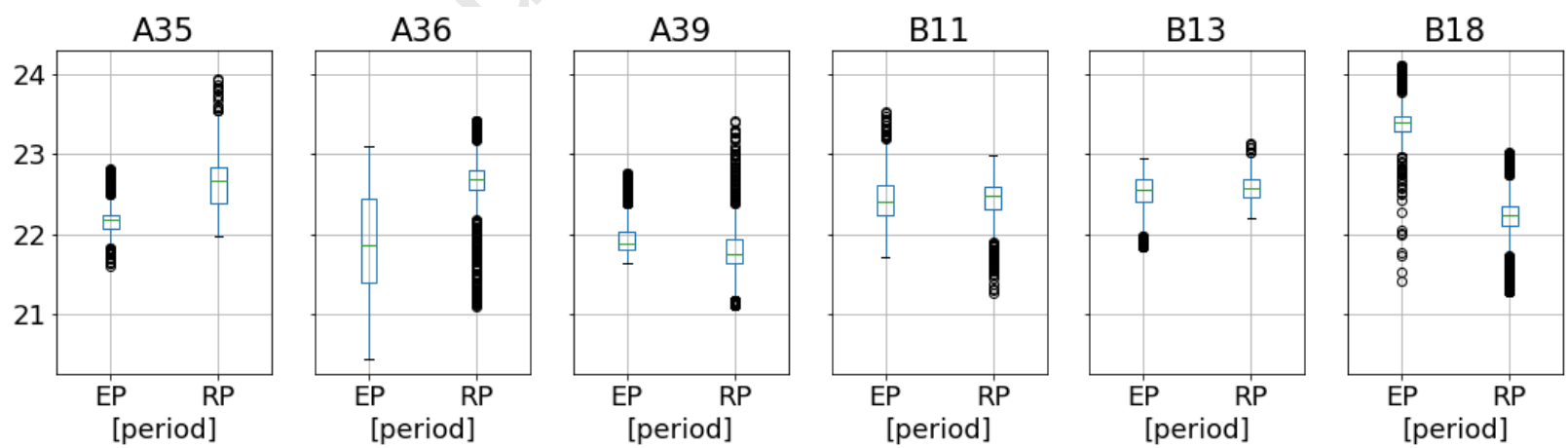

Figure 8: Box plot of lumped temperatures in experiment period (EP) and reference period (RP) for control group of apartments

In order to make a comparison across apartments and periods we calculate the lumped indoor $\mathrm{RMS}_{i}^{1}$ for each apartment using equations (5) and (7). We find that in general the errors are low, all below $1^{\circ} \mathrm{C}$ with average values for both periods below $0.4^{\circ} \mathrm{C}$. An effect of the experiment on the indoor temperature would be indicated by an increase in error but 
Table 4: Total energy consumption and change in percent from reference period results

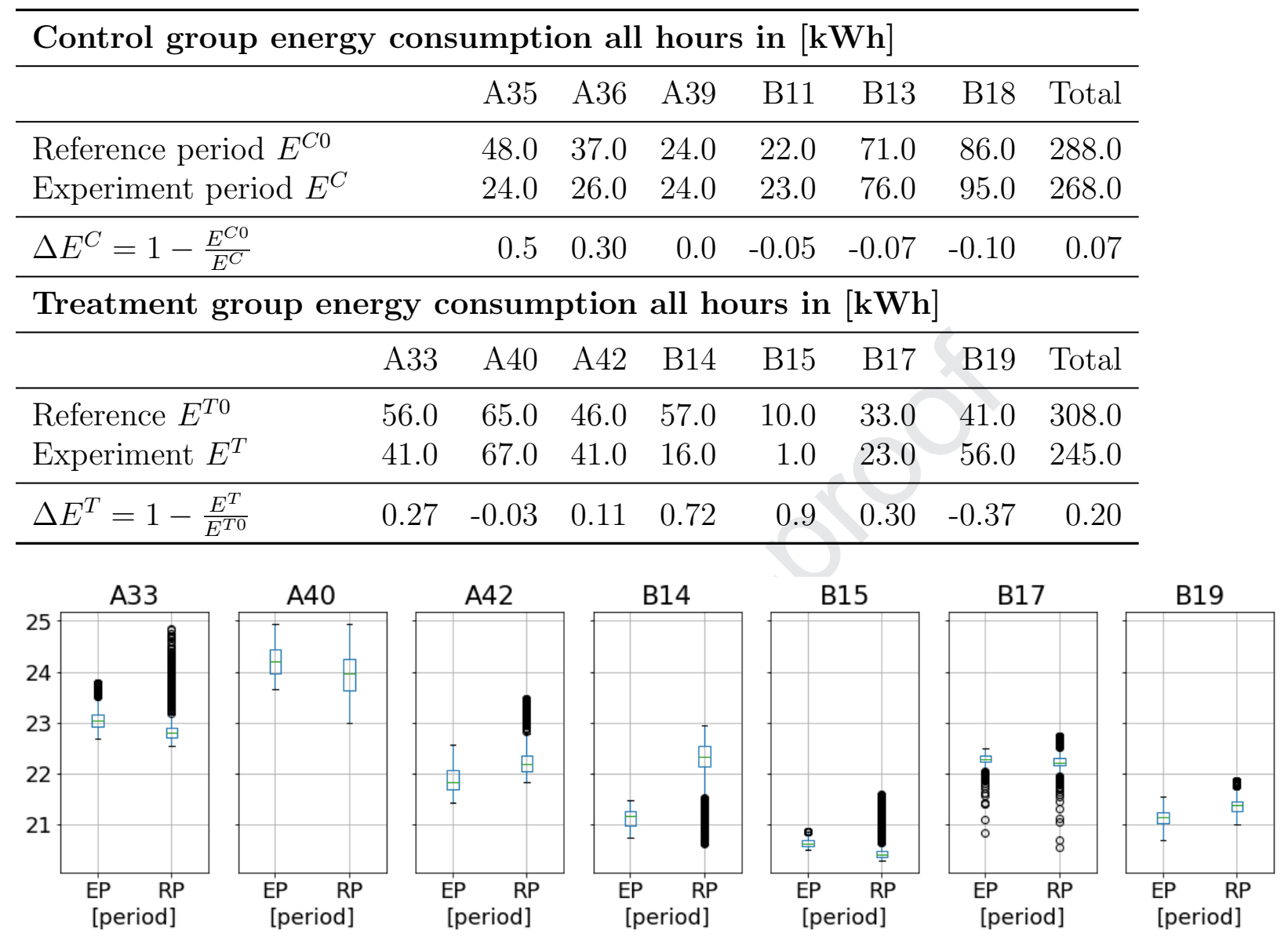

Figure 9: Box plot of lumped temperatures in experiment period (EP) and reference period (RP) for treatment group of apartments

as can be observed from Table 5 the $\widetilde{\mathrm{RMS}_{i}^{1}}$ of the Treatment group actually drops in the Experiment period, where for the control group the error is essentially unchanged, giving strength to interpretation that the experiment had a small effect on the indoor temperature.

\section{Conclusion}

In this study we demonstrated how to remotely control the heating systems in individual rooms in real-world apartments to lower heating demand in morning peak hours. The effect of the reduction on the energy consumption is compared to a reference group of similar apartments in a reference period of similar days. We found that $85 \%$ reduction was achieved by the simple control strategy. The ideal case of $100 \%$ reduction during the penalty period is thus not achieved. The control of the bathrooms where omitted as explained in subsection 3.2 due to user constraints. This could account for some of this deviation from the ideal case. Another cause could be delays or mechanical problems in the closing of 
Table 5: Control group RMSE of temperature deviations during peak hours 06.00-12.00

\begin{tabular}{|c|c|c|c|c|c|c|c|c|}
\hline \multicolumn{9}{|c|}{ Control group RMSE of temperature deviations during peak hours 06.00-12.00 } \\
\hline & & A35 & A36 & A39 & B11 & B13 & B18 & Mean \\
\hline Reference & & 0.271 & 0.458 & 0.260 & 0.330 & 0.191 & 0.446 & 0.326 \\
\hline Experiment & & 0.227 & 0.704 & 0.243 & 0.288 & 0.286 & 0.292 & 0.340 \\
\hline \multicolumn{9}{|c|}{ Treatment group RMSE of temperature deviations during peak hours $06.00-12.00$} \\
\hline & A33 & A40 & A 42 & B14 & B15 & B17 & B19 & Mean \\
\hline Reference & 0.155 & 0.464 & 0.199 & 0.869 & 0.160 & 0.117 & 0.264 & 0.318 \\
\hline Experiment & 0.261 & 0.437 & 0.257 & 0.203 & 0.089 & 0.139 & 0.282 & 0.238 \\
\hline
\end{tabular}

valves. These types of phenomena are hard to account for in simulations and thus highlights the value of conducting real world trials to verify and validate the results obtained from previous simulation-based studies. The results confirmed the potential of the automated buildings to reduce the energy consumption during peak hours with little disturbance on indoor temperatures. Such controls could thus help the district heating systems reduce the use of fossil fuels and the investment in costly central storage solutions.

The verification of the effects of the experiments from the grid side and the inclusion of advanced control methods such as predictive controls and economic MPC are left for future studies. By applying advanced control methods, multiple objectives could be simultaneously pursued, and increased flexibility might be achievable along with lower variations in the indoor temperature by accounting for the impact of solar gains, outdoor temperature and internal gains in the controls. Additionally, the method proposed could be extended and applied to e.g. cooling systems or other forms of heating systems like heat pumps or direct electrical heating. Further, the results of the experiment can be used in formulating noise and uncertainty models that in turn can be used to improve on the accuracy and reliability of simulations-based studies 


\section{Acknowledgement}

The authors would like to thank the EUDP for funding through the EnergyLab Nordhavn project (EUDP 64015-0055).

\section{References}

[1] S. Chu, Y. Cui, N. Liu, The path towards sustainable energy (2016). doi:10.1038/nmat4834.

[2] M. Mazzucato, G. Semieniuk, Financing renewable energy: Who is financing what and why it matters, Technological Forecasting and Social Change 127 (2018) 8-22. doi:10.1016/J.TECHFORE. 2017.05. 021 .

URL https://www .sciencedirect.com/science/article/pii/S0040162517306820

[3] International Energy Agency (IEA), International Partnership for Energy Efficiency Cooperation (IPEEC), Building Energy Performance Metrics Supporting Energy Efficiency Progress in Major Economies, Tech. rep. (2015).

URL https://www . iea.org/reports/building-energy-performance-metrics

[4] P. Capros, A. Vita, N. Tasios, D. Papadopoulos, P. Siskos, e. a. Apostolaki E, EU energy, transport and GHG emissions: trends to 2050 - reference scenario 2013 (2014).

URL https://ec.europa.eu/transport/sites/transport/files/media/publications/doc/ trends-to-2050-update-2013.pdf

[5] S. Frederiksen, S. Werner, District heating and cooling, Studentlitteratur, 2013.

[6] C. Ordoudis, P. Pinson, J. M. Morales, An Integrated Market for Electricity and Natural Gas Systems with Stochastic Power Producers, European Journal of Operational Research 272 (2) (2019) 642-654. doi:10.1016/J.EJOR.2018.06.036.

URL https://www.sciencedirect.com/science/article/abs/pii/S037722171830571X

[7] E. A. M. Cesena, P. Mancarella, Operational optimization and environmental assessment of integrated district energy systems, in: 2016 Power Systems Computation Conference (PSCC), IEEE, 2016, pp. 1-7. doi:10.1109/PSCC.2016.7540972. URL http://ieeexplore.ieee.org/document/7540972/

[8] H. Lund, S. Werner, R. Wiltshire, S. Svendsen, J. E. Thorsen, F. Hvelplund, B. V. Mathiesen, 4th Generation District Heating (4GDH). Integrating smart thermal grids into future sustainable energy systems. (2014). doi:10.1016/j.energy.2014.02.089.

[9] H. Lund, P. A. Østergaard, D. Connolly, B. V. Mathiesen, Smart energy and smart energy systems (2017). doi:10.1016/j.energy.2017.05.123.

[10] K. Hansen, C. Breyer, H. Lund, Status and perspectives on 100\% renewable energy systems, Energy 175 (2019) 471-480. doi:10.1016/j.energy .2019.03.092.

URL https://linkinghub.elsevier.com/retrieve/pii/S0360544219304967

[11] Directive (EU) 2018/844 of the European Parliment and of the council of 30 May 2018 amending Directive 2010/31/EU on the energy performance of buildings and Directive 2012/27/EU on energy efficiency, Tech. rep.

URL https://eur-lex.europa.eu/legal-content/EN/TXT/PDF/?uri=CELEX :32018L0844\&f rom=EN

[12] G. T. Costanzo, G. Zhu, M. F. Anjos, G. Savard, A System Architecture for Autonomous Demand Side Load Management in Smart Buildings, IEEE Transactions on Smart Grid 3 (4) (2012) 2157-2165. doi:10.1109/TSG.2012.2217358.

URL http://ieeexplore .ieee.org/document/6376273/

[13] P. Pinson, T. Baroche, F. Moret, T. Sousa, E. Sorin, S. You, The Emergence of Consumer-centric Electricity Markets, Tech. rep.

URL http://pierrepinson.com/docs/pinsonetal17consumercentric.pdf

[14] K. Foteinaki, R. Li, A. Heller, C. Rode, Heating system energy flexibility of low-energy residential buildings, Energy \& Buildings 180 (2018) 95-108. doi:10.1016/j.enbuild.2018.09.030 URL https://doi.org/10.1016/j.enbuild.2018.09.030 
[15] H. Cai, C. Ziras, S. You, R. Li, K. Honoré, H. W. Bindner, Demand side management in urban district heating networksdoi:10.1016/j.apenergy .2018.08.105.

[16] P. Kohlhepp, H. Harb, H. Wolisz, S. Waczowicz, D. Müller, V. Hagenmeyer, Large-scale grid integration of residential thermal energy storages as demand-side flexibility resource: A review of international field studies, Renewable and Sustainable Energy Reviews 101 (February 2018) (2019) 527-547. doi: 10.1016/j.rser.2018.09.045

[17] G. Le Ray, E. M. Larsen, P. Pinson, Evaluating price-based demand response in practice - with application to the EcoGrid EU Experiment, IEEE Transactions on Smart Grid (2016) 1-1doi: 10.1109/TSG.2016.2610518.

URL http://ieeexplore.ieee.org/document/7571156/

[18] M. Alam, A. A. Panagopoulos, A. Rogers, N. R. Jennings, J. Scott, Applying extended kalman filters to adaptive thermal modelling in homes, in: Proceedings of the 1st ACM Conference on Embedded Systems for Energy-Efficient Buildings - BuildSys '14, ACM Press, New York, New York, USA, 2014, pp. 214-215. doi:10.1145/2674061.2675041.

URL http://dl .acm.org/citation. cfm?doid=2674061.2675041

[19] M. Hu, F. Xiao, J. B. Jørgensen, R. Li, Price-responsive model predictive control of floor heating systems for demand response using building thermal mass, Applied Thermal Engineering 153 (February) (2019) 316-329. doi:10.1016/j.applthermaleng.2019.02.107.

[20] C. Finck, R. Li, W. Zeiler, Economic model predictive control for demand flexibility of a residential building, Energydoi:10.1016/j.energy.2019.03.171.

URL https://linkinghub.elsevier .com/retrieve/pii/S0360544219305912

[21] Y. Zong, G. M. Böning, R. M. Santos, S. You, J. Hu, X. Han, Challenges of implementing economic model predictive control strategy for buildings interacting with smart energy systems, Applied Thermal Engineering 114 (2017) 1476-1486. doi:10.1016/J.APPLTHERMALENG .2016.11.141.

URL https://www.sciencedirect.com/science/article/pii/S1359431116334871

[22] E. Zilio, K. Foteinaki, P. Gianniou, C. Rode, Impact of Weather and Occupancy on Energy Flexibility Potential of a Low-energy Building, Building Simulation 2017.

URL http://www.ibpsa.org/proceedings/BS2017/BS2017_274.pdf

[23] T. Sweetnam, C. Spataru, M. Barrett, E. Carter, Domestic demand-side response on district heating networks, Building Research and Information 47 (4) (2019) 330-343. doi:10.1080/09613218.2018. 1426314

[24] R. A. Lopes, A. Chambel, J. Neves, D. Aelenei, J. Martins, A Literature Review of Methodologies Used to Assess the Energy Flexibility of Buildings, Energy Procedia 91 (2016) 1053-1058. doi:10.1016/J. EGYPRO.2016.06.274. URL https://www.sciencedirect.com/science/article/pii/S1876610216303745

[25] R. G. Junker, A. G. Azar, R. A. Lopes, K. B. Lindberg, G. Reynders, R. Relan, H. Madsen, Characterizing the energy flexibility of buildings and districts, Applied Energy 225 (2018) 175-182. doi:10.1016/J.APENERGY.2018.05.037. URL https://wWw.sciencedirect.com/science/article/pii/S030626191830730X?via\%3Dihub

[26] L. Liu, L. Fu, Y. Jiang, A new "wireless on-off control" technique for adjusting and metering household heat in district heating system, Applied Thermal Engineering 36 (1) (2012) 202-209. doi:10.1016/j . applthermaleng.2011.11.040.

URL http://dx.doi.org/10.1016/j.applthermaleng.2011.11.040

[27] S. Kärkkäinen, K. Sipilä, L. Pirvola, Demand side management of the district heating systems, Vtt.Fi. URL http://www.vtt.fi/inf/pdf/tiedotteet/2004/T2247.pdf

[28] EnergyLab Nordhavn - official project web page.

URL http://www . energylabnordhavn. com/

[29] Sundmolehusene - på Østerbro og lige ud til Øresund

URL https://sundmolehusene.dk/

[30] The Danish transport and construction agency, Danish Building Regulations 2015 (2015). URL https://historisk. bygningsreglementet.dk/file/591081/br15_english.pdf 
[31] L. Sarran, M. Herget Christensen, C. A. Hviid, A. M. Radoszynski, C. Rode, P. Pinson, Data-driven study on individual occupant comfort using heating setpoints and window openings in new low-energy apartments - preliminary insights, E3S Web of Conferences 111 (2019) 04063. doi:10.1051/e3sconf/ 201911104063 .

[32] K. Foteinaki, R. Li, A. Heller, M. Herget Christensen, C. Rode, Dynamic thermal response of lowenergy residential buildings based on in-wall measurements, E3S Web of Conferences 111 (2019) 04002. doi:10.1051/e3sconf/201911104002.

[33] KNX standard Version 2.1

URL https://my.knx.org/en/shop/knx-specifications

[34] R. A. Light, Mosquitto: server and client implementation of the MQTT protocoldoi:10.21105/joss. 00265

URL https://www.theoj.org/joss-papers/joss.00265/10.21105.joss.00265.pdf

[35] M. Herget Christensen, Code to control floor heating setpoints according to schedules, Source code on Zenododoi: 10.5281/zenodo.3600114.

URL https://zenodo.org/record/3600114

[36] J. H. Eto, On using degree-days to account for the effects of weather on annual energy use in office buildings, Energy and Buildings 12 (2) (1988) 113-127. doi:10.1016/0378-7788(88)90073-4 URL https://www.sciencedirect.com/science/article/pii/0378778888900734

[37] L. Lundström, Adaptive Weather Correction of Energy Consumption Data, Energy Procedia 105 (2017) 3397-3402. doi:10.1016/J.EGYPRO.2017.03.778.

URL https://www.sciencedirect.com/science/article/pii/S1876610217308469

[38] L. Lundström, F. Wallin, Heat demand profiles of energy conservation measures in buildings and their impact on a district heating system, Applied Energy 161 (2016) 290-299. doi:10.1016/j . apenergy . 2015.10.024.

URL https://linkinghub.elsevier.com/retrieve/pii/S0306261915012519

[39] S. O. Jensen, A. Marszal-Pomianowska, R. Lollini, W. Pasut, A. Knotzer, P. Engelmann, A. Stafford, G. Reynders, IEA EBC Annex 67 Energy Flexible Buildings, Energy and Buildings 155 (2017) 25-34. doi:10.1016/j.enbuild.2017.08.044

URL http://linkinghub.elsevier.com/retrieve/pii/S0378778817317024 
- Control of individual floor heating loops respects user preferences

- Cloud connected home automation systems used to provide energy systems services

- High thermal mass of low energy buildings allow flexibility in energy consumption

- No loss of comfort for residents or increase in overall energy consumption 


\section{Declaration of interests}

$\bigotimes$ The authors declare that they have no known competing financial interests or personal relationships that could have appeared to influence the work reported in this paper.

$\square$ The authors declare the following financial interests/personal relationships which may be considered as potential competing interests:

Morten Herget Christensen

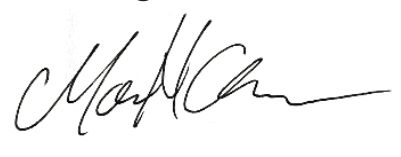

Rongling Li

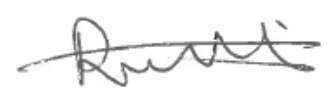

Pierre Pinson

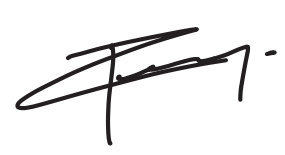

